Título artículo / Títol article:

Prácticas docentes, condiciones de enseñanza y oportunidades de aprendizaje inicial de la lengua escrita

Autores / Autors

Isabel Ríos García, Pilar Fernández, Isabel Gallardo

Revista:

Cultura y Educación. Revista de teoría, investigación y práctica

Versión / Versió:

Preprint

Cita bibliográfica / Cita bibliogràfica (ISO 690):

RÍOS GARCÍA, Isabel; FERNÁNDEZ Pilar; GALLARDO Pilar. Prácticas docentes, condiciones de enseñanza y oportunidades de aprendizaje inicial de la lengua escrita. Cultura y Educación. Revista de teoría, investigación y práctica, 2012 , vol. 24, no 4, p. 435-447 (13).

url Repositori UJI:

http://hdl.handle.net/10234/63526 


\section{PRÁCTICAS DOCENTES, CONDICIONES DE ENSEÑANZA Y OPORTUNIDADES DE APRENDIZAJE INICIAL DE LA LENGUA ESCRITA}

\section{TEACHING PRACTICES, TEACHING CONDITIONS AND CHANCES FOR}

\section{EARLY LITERACY LEARNING}

Isabel Ríos ${ }^{1}$, Universitat Jaume I; Pilar Fernández ${ }^{2}$, Universidad Complutense de Madrid; Isabel Gallardo ${ }^{3}$, Universitat de Valencia

\section{Resumen}

Formatat: Justificada

El objetivo de este trabajo es explorar la relación entre las prácticas utilizadas por los docentes para enseñar a leer y a escribir en el inicio de la escolaridad y las oportunidades de aprendizaje. Se realiza un análisis cualitativo de una serie de tareas llevadas a cabo en aulas donde desarrollan tres tipos de prácticas diferentes - instruccionales, multidimensionales y situacionales -. Los resultados muestran que estas prácticas difieren en cuanto al tipo de tareas llevadas a cabo; en cuanto a las dinámicas desarrolladas para llevarlas a cabo; en cuanto a los contenidos de lectura y escritura trabajados y en cuanto a los materiales utilizados. Muestran, asimismo, que determinados rasgos que distintos autores relacionan con un mayor nivel de eficacia docente y con la potenciación de oportunidades de aprendizaje se observaron con más frecuencia en dos de las aulas.

Palabras clave: enseñanza lengua escrita, interacción, prácticas docentes, análisis de situaciones de aula

\section{Abstract}

The aim of this paper is to explore the relationship between the practices used by teachers to teach to read and write at the beginning of schooling and learning opportunities. A qualitative 
analysis of a number of tasks carried out by teachers who used three types of different practices - instructional, multidimensional and situational - is performed. The results show that these practices differ as to the type of tasks carried out; as for classroom dynamics displayed; with regard to the contents of early literacy worked and in terms of materials used. It is also showed that in two of the three classrooms certain traits that various authors have associated with a higher level of teaching effectiveness as well as with enhancing learning opportunities were more often seen.

Key words: literacy teaching, interaction, classroom practices, classroom analysis

\section{Correspondencia con autoras:}

Isabel Ríos: Dep. de Educación. Universitat Jaume I. 12071 Castelló. Tlf: 964729796 . Fax: 964729264. rios@edu.uji.es

Pilar Fernández: fernandez.pilar@telefonica.net

| Isabel Gallardo: DOE. Universitat de València. Av. Blasco Ibáñez 30., Facultat de Filosofía i Ciències de l’Educació. Valencia. Tlf: 963864602. Fax. 963864971 isabel.gallardo@uv.es

El presente trabajo forma parte del proyecto de investigación Las condiciones del aprendizaje inicial de la lengua escrita: influencia de las prácticas vigentes en el aula y de los conocimientos previos de los alumnos (SEJ 2006/05292), subvencionado por el MEC en el Plan Nacional de I+D+I (2004-2007), cuyo objetivo principal es determinar las condiciones de aprendizaje de la lengua escrita identificando la influencia relativa de las prácticas docentes y de las competencias iniciales del alumnado.

| Equipo investigador: I. P. Liliana Tolchinsky (Universitat de Barcelona), M. Fons, C. Buisán, C. Alba (Universitat de Barcelona), M. Bigas (Universitat Autònoma de Barcelona), C. Glez. Landa, P. Fernández, T. Chamorro, I. Ga. Parejo (Universidad Complutense de Madrid), L. Barrio (Universidad de Valladolid), T. Llamazares, $\mathrm{M}^{\mathrm{a}} \mathrm{D}$. Alonso-Cortés (Universidad de León), I. Ríos (Universitat Jaume I), P. Ribera, I. Gallardo (Universitat de València), C. Barragán y M Ma Medina (Universidad de Almería), X. A. Glez. Riaño, L. Díaz (Universidad de Oviedo), S. Sánchez (Universidad de Cantabria), M. Bilbatua, G. Orbea (Mondragón Unibertsitatea). 


\section{Introducción}

La necesidad de conocer las condiciones en que se enseña a leer y a escribir en las aulas surge de la insatisfacción, generalizada en el ámbito social y académico, por los resultados obtenidos en los niveles de enseñanza obligatoria (ver informes PISA y PIRLS, 2006).

La investigación educativa ha ofrecido ya numerosos trabajos sobre los procesos y condiciones implicados en las situaciones de lectura y escritura (Wells, 1988; 2000; Bereiter y Scardamalia, 1992; Hayes y Flower, 1986). Desde la perspectiva sociocultural se defiende que la actividad didáctica no tiene sentido si no se enmarca en la actividad social (Bruner, 1997; Olson, 2004; Graves, 1983; Camps y Ribas, 2000; Tolchinsky, 1993; 2001; Fons, 1999; Fernández 2007, entre otros). Esta perspectiva considera las actividades humanas (como lo son leer y escribir) fruto de la interacción en contextos sociales determinados, que conllevan acciones encaminadas a comunicarse y aprender.

Una mirada integradora de lo cognitivo y lo sociocultural ofrece como resultado trabajos de gran importancia para la temática que nos ocupa. Sin embargo, el conocimiento que se ha desarrollado desde los estudios sobre la génesis de la lengua escrita (Ferreiro y Teberosky, 1979 y numerosos trabajos posteriores); a partir de las aportaciones de la psicología históricocultural (Vigotsky, 1973); y sobre la consideración de las actividades letradas en situaciones sociales determinadas que dan lugar a los géneros (Bajtín, 1982) no parece haberse incorporado a la práctica docente.

Por otro lado, aunque existe un amplio consenso en cuanto a que el aprendizaje inicial de la lengua escrita no termina con el aprendizaje del código sino que requiere desde el principio usos cada vez más complejos como registrar, comunicar y transformar conocimientos sobre la lengua escrita (Camps, 1994; Teberosky y Tolchinsky, 1995; Solé et al., 2005), estudios que han analizado prácticas educativas (Altava, Gimeno, Pérez, Ríos y Gallardo, 2006; Dolz y Plane, 2008) muestran concepciones subyacentes diferentes o que enfatizan otros aspectos. 
Encontramos también estudios que reclaman dirigir la mirada hacia los resultados de aprendizaje poniéndolos en relación con el tipo de prácticas utilizadas para enseñar a leer y escribir (Morrow y Schickedanz, 2006; Morrow y Gambrell, 2006). Especialmente interesante es el estudio de Wharton-MacDonald, Pressley y Hampston, (1998) quienes mostraron a través de la observación de nueve maestros que las tareas conllevan diferente grado de utilidad para la alfabetización de los alumnos. Destacaron que se obtenían mejores resultados en las aulas en las que se dedicaba una gran parte del tiempo a producir y leer textos. En estas aulas, por otro lado, el mensaje de "puedo ser lector" se encontraba subyacente en la actividad cotidiana. La lectura de estos alumnos, en su mayoría, superaba los supuestos niveles del grado en el que se encontraban. Las experiencias de lectura y escritura reales y la enseñanza explícita de habilidades ocupaban un tiempo equilibrado; se accedía mejor a las actividades letradas; y los alumnos, al finalizar el curso escolar, eran más capaces de escribir, de forma autónoma, textos largos, con ideas más claramente expuestas y con mejor ortografía. El ambiente era positivo y la actividad de leer y escribir se encontraba presente de formas muy diversas. Además, estaban más motivados para leer y escribir y para hacerlo bien y con una mayor capacidad de lectura por decisión propia. El aprendizaje de los contenidos escolares conllevaba una actividad de lectura y escritura y los maestros prestaban las ayudas necesarias para que la tarea a la que se enfrentaban fuera exitosa. La actividad escolar de estas aulas era, también, de alto nivel. Es decir, los niños se enfrentaban a tareas complejas y difíciles, para las que requerían la ayuda del docente.

Pressley et al. (2001), por su parte, exploraron y determinaron qué factores tienen un efecto positivo en los resultados de los niños. Profundizaron en los comportamientos y las propuestas didácticas de los docentes para deducir las características de las prácticas más o menos eficaces y destacaron, por ejemplo, la importancia de que los niños lean y escriban mucho; de que se provoquen procesos de escritura de orden superior; de que se aprovechen 
los emergentes y de que se ayude a los niños a hacer tareas más allá de sus propias posibilidades autónomas.

La evaluación de los resultados obtenidos por los aprendices se basó en la observación de actividades de alfabetización y en la lectura y escritura de los alumnos. Se tuvo en cuenta el tiempo dedicado a las actividades de lectura y escritura, al nivel de comprensión de textos y la calidad de los textos producidos. Los resultados mostraron que en las aulas más eficaces la mayoría de los alumnos (un 90\%) pasaban el 90\% del tiempo escolar realizando actividades de lectura y escritura. El desempeño de estos niños en lectura les permitía leer libros de varias oraciones por página o de capítulos, al final del primer año de escolaridad (equivalente a nuestro primer año de educación primaria). Sus composiciones escritas eran de varias páginas, escribían con coherencia y puntuaban, usaban las mayúsculas y la ortografía inventada era escasa. Sin embargo, en las aulas con prácticas menos eficaces, menos del $9 \%$ de los niños dedicaban un $90 \%$ del tiempo escolar a leer y escribir. Al final del año escolar se observaba que leían unas pocas palabras por página y sus textos escritos eran cortos, de un par de frases.

A partir de estos resultados los autores propusieron una serie de rasgos para la identificación de los profesores más efectivos: el horario de clase; la naturaleza de las actividades de lectura y escritura y los tipos de texto que se leían y escribían; el uso de emergentes; las conexiones de la lectura y la escritura con el currículum y los procedimientos para la gestión del aula. Se determinó que el profesor más eficaz maneja la clase de forma excelente. En su aula, el ambiente y la dinámica es positiva y de colaboración. El tiempo y las tareas de desciframiento y las de composición y lectura de textos están equilibrados y se atiende a la lectura de pistas, anticipación, contextualización de los significados, etc. Las actividades de lectura y escritura se realizan en diversos contextos, de forma colectiva, autónoma, en colaboración, etc. Se usan libros cada vez más difíciles y se les ofrecen a los niños oportunidades para planificar, 
redactar, revisar y publicar sus textos. Los retos que se les proponen son asequibles gracias a la cantidad de andamiajes que se les prestan y hay un incremento continuo de las expectativas que comparten profesores y alumnos.

En otra investigación, Castells (2006; 2009a) comparó los conocimientos de los niños que han recibido instrucción en aulas en las que se llevan a cabo actividades ligadas a los métodos de enseñanza de la lectura y la escritura analítico, sintético y analítico-sintético. Observó que las aproximaciones analítica y analítico-sintética favorecen que los niños desarrollen estrategias dirigidas a comprender y destacó la importancia que esto tiene al realizar actividades de lectura más complejas que la oralización de un texto. Concluyó que las prácticas analíticas o analítico-sintéticas comportan tareas de lectura que van más allá del desciframiento para adentrarse en la extracción de significado. Comportan también una relación con los géneros y los diferentes textos sociales que enfrentan a los niños a actividades de comunicación más allá del uso y aprendizaje del código como tarea esencial en el proceso de alfabetización.

En la línea de estas investigaciones, nuestro estudio tiene como objetivo explorar la relación entre las prácticas utilizadas por los docentes para enseñar a leer y a escribir en Infantil-5 años y en $1^{\circ}$ de Primaria y las oportunidades de aprendizaje. Tolchinsky, Ribera y García-Parejo (en este número) mencionan que no han encontrado relación entre las distintas prácticas detectadas en las aulas y los logros de los niños al finalizar $1^{\circ}$ de Primaria. Sus datos fueron obtenidos mediante la aplicación, fuera del aula, de protocolos de evaluación, de modo que hacen referencia a los productos del aprendizaje. El presente trabajo, enmarcado en el mismo proyecto de investigación ${ }^{4}$, pretende abordar dicha relación atendiendo a los procesos de aprendizaje que se ponen en marcha en el contexto de aula. Para ello, hemos realizado un análisis cualitativo de una serie de tareas ${ }^{5}$ llevadas a cabo en aulas que desarrollan tres tipos de prácticas diferentes (González, Buisán, y Sánchez, 2009; Fons y Buisán, en este número): 
prácticas instruccionales, centradas fundamentalmente en actividades de enseñanza explícita del código y atención frecuente a los resultados del aprendizaje; prácticas situacionales, decantadas hacia actividades de escritura autónoma, aprovechamiento de situaciones emergentes y no tan frecuente preocupación por los resultados; y prácticas multidimensionales, en las que destaca la escritura autónoma y el aprovechamiento de emergentes, pero hay una preocupación frecuente por los productos de aprendizaje.

\section{Método}

Participantes

Los participantes en este trabajo son las maestras y el alumnado de tres aulas (A, B y C). Estas aulas forman parte de un conjunto de 10 (5 aulas de Infantil-5 años y 5 de $1^{\circ}$ de Educación Primaria) que a lo largo de dos cursos escolares visitamos con el fin de recopilar los datos necesarios para llevar a cabo la investigación marco. Estas aulas fueron seleccionadas porque en cada una de ellas se había identificado uno de los perfiles de práctica docente previamente definidos: las prácticas del Aula A (Infantil-5 años, centro público, 25 alumnos) eran prácticas situacionales; las prácticas del Aula B ( $1^{\circ}$ de Primaria, centro concertado, 26 alumnos), multidimensionales, y las prácticas del Aula $\mathrm{C}\left(1^{\circ}\right.$ de Primaria, centro concertado, 27 alumnos) eran prácticas de tipo instruccional.

\section{Procedimiento}

Para recopilar los datos, en cada aula y en cada curso se realizaron como mínimo cuatro observaciones. La primera observación tenía como finalidad conocer y describir espacios, horarios, agrupamientos de alumnos, tipos de actividades y materiales. Después, se realizaron observaciones tanto de las sesiones que las maestras planificaban específicamente para aprender a leer y/o a escribir (observaciones específicas) como de sesiones no 
específicamente planeadas para la enseñanza de la lectura y/o la escritura (observaciones parciales).

En cada sesión de observación, con una duración media de 1 h.40 minutos, se observó un conjunto de tareas. El número de tareas observadas en cada una de las aulas figuran en la Tabla I:

Tabla I aprox. aquí

En el aula con prácticas de tipo instruccional se realizó un menor número de observaciones debido a que las prácticas de estas aulas son reiterativas y tienen dinámicas similares en las diferentes sesiones, por lo que un acceso tan frecuente no resultó necesario.

Las notas de campo recogidas durante las observaciones se volcaron luego en unas tablas de registro de $\operatorname{tareas}^{6}$ que constituyen el corpus de datos para el análisis cualitativo, que ha consistido en estudiar la actividad de cada aula en función de cuatro categorías: las características de las tareas que se llevan a cabo; las dinámicas de aula; los contenidos de enseñanza implicados; los materiales empleados en las tareas y el uso que se les da. Estas categorías se concretan en los aspectos que se enumeran en la Tabla II.

Tabla II aprox. aquí

\section{Resultados}

En la Tabla III se presentan los resultados obtenidos del estudio del corpus, a partir de las categorías de análisis establecidas.

Tabla III aprox. Aquí 
A continuación se explican dichos datos, describiendo cómo eran las tareas y las dinámicas que se llevaban a cabo en cada aula y cuáles eran los contenidos de enseñanza y los materiales que se utilizaban. El análisis se ilustra con ejemplos de tareas prototípicas.

\section{Aula A: prácticas de tipo situacional}

Respecto a la dinámica del aula A se observó que la mayoría de las tareas eran largas, complejas y con sentido para los niños. El trabajo se planteaba en colaboración o de forma colectiva; se escribían textos funcionales y de uso social. Se aprovechaban los emergentes (situaciones que surgen en el aula de forma inesperada) y se reorientaba la actividad en función de los sucesos que se producían. La maestra incitaba al razonamiento y a las explicaciones sobre lo que se hacía y por qué se hacía. Las tareas requerían esfuerzo pero se trabajaba desde el interés despertado en los niños, con ayuda de la maestra. Estaban estructuradas pero eran flexibles y se adecuaban a cada persona en función de los procesos de aprendizaje u otros aspectos personales. En las actividades colectivas, la maestra pedía la participación de los niños para elaborar el contenido del texto y la forma de escribirlo, así como para leer o anticipar los significados. Los errores eran fuente de reflexión para el grupo y para quien los cometía.

Los contenidos de enseñanza que se abordaron fueron: función de la lectura y la escritura, léxico, familiaridad con los textos, denominación de aspectos de organización textual, escritura, identificación de palabras o lectura, nombre y valor sonoro de las letras.

Se usaron materiales diversos para leer y escribir y se aprovecharon los soportes que llegaban al aula o de uso social: carteles, periódicos, libros de conocimientos.

Para ilustrar el análisis, ofrecemos un ejemplo de tarea de práctica situacional. Consistió en la elaboración colectiva de un texto en la pizarra, lectura del mismo y posterior copia. Se trata 
de una nota para los padres, para convocarles a la participación en la Fiesta de S. Antonio (la Matxà de Sant Antoni)"”.

(La maestra trae a la clase una situación social e intenta interesar a los niños sobre los hechos y la importancia de comunicar por escrito. A partir de la conversación previa, se guía a los niños en el proceso de elaboración del texto. Los niños participan libremente y la maestra les va dando la palabra. Recoge las aportaciones relevantes para convertirlas en el texto de la nota que dirigirán a sus padres.)

M. Tenim que fer un paperet per a que els pares i mares sàpiguen lo de la matxà 'Tenemos que hacer un papelito para que los padres y las madres sepan lo de la matxá'

(...)

N1. ...pero mi madre no sabe dónde está...

M. Mira! Què acaba de dir la nostra amigueta Nuria... que no sabrà la mare... Anem a escriure per a dir el dia, l'hora, el lloc... Anem a pensar... Si no ho aclarim bé no podrem anar..

'Mira! Qué acaba de decir nuestra amiguita Nuria... que no sabrá la madre... Vamos a escribir para decir el día, la hora, el lugar... Vamos a pensar... Si no lo aclaramos bien no podremos ir'

(Con el texto que van proponiendo, M pide que le digan las letras de cada palabra, como por ejemplo vesprada, 'tarde')

$\mathrm{N} 2$ dicta las letras: la be baixa, la $e$, la esa, la pe, la erre, la $a$, la $d e$, la $a \ldots$

N1 corrige: falta la $e$ (La M le da un beso)

(Cada vez que tienen un fragmento escrito $\mathrm{M}$ les hace leer a coro o a veces pregunta a un niño concreto que lea $<$ El dimecres a les 7 de la vesprada>)

$(\ldots)$

M. Ara ve una molt difícil que sona com la esa (se refiere a la ç, de la palabra plaça) 'Ahora viene una muy difícil que suena como la ese'

Ns (a coro) ce trencada 'ce con cedilla'

(Dictan todas las palabras con sus letras)

M. Matxà

N3: la che

M. Com s'escriu la che?

$\mathrm{N} 3$ : la equis

$(\ldots)$

M. S'ens ha oblidat una cosa. A qui li ho donem? 'se nos ha olvidado una cosa. ¿A quién se lo damos?' 
N3. Als pares. 'A los padres'

M. Posarem: pare i pare. 'Pondremos: padre y madre'

(M lo escribe y ya no les pide dictarlo a ellos. El texto definitivo queda como sigue:

<PARE I MARE:

EL DIMECRES A LES 7 DE LA VESPRADA A LA PLAÇA DELS LLUÏSOS ANIREM A LA MATXADA DELS ANIMALETS DE SANT ANTONI.>

A continuación leen colectivamente el texto: la maestra lee siguiendo con la mano cada fragmento significativo del texto, no cada palabra. Algunos niños la acompañan en la lectura y otros van siguiendo el texto con la mirada. Luego pide a una niña que lea sola, que lee correctamente).

(Los niños escriben la nota copiando de la pizarra).

M. Ah! I ací baix ficareu cadascú el vostre nom. Ah! La lletra te que estar molt bé. Molt entenedora, eh? ‘¡Ah! Y aquí abajo pondréis cada uno vuestro nombre. ¡Ah! La letra tiene que estar muy bien. Muy comprensible, ¿eh?’

(Algunos niños escriben sin mirar continuamente a la pizarra porque recuerdan el texto que han construido y son capaces de escribirlo de forma autónoma).

Aula B: prácticas de tipo multidimensional

En esta aula hubo diversidad de tareas. En cuanto a la dinámica de aula, se observó que el trabajo se planteaba en gran grupo o individual. La maestra creaba un clima de seguridad y afecto y apoyaba, continuamente, a los niños que más la necesitaban. En las actividades de gran grupo, procuraba que participaran todos los niños.

Hubo diversidad de tareas. Se seguía un método de enseñanza de la lectura y la escritura del mercado editorial pero se realizaban además otras tareas que la maestra proponía o que surgían, relacionadas con la vida del aula o de las familias. Además se utilizaban algunos textos funcionales y de uso social. Algunas tareas eran largas, complejas y significativas para los niños; otras, las del método, eran cortas y con poco sentido y funcionalidad para los niños. El trabajo con el método de lectura ocupaba mucho tiempo. En alguna ocasión se aprovechaban emergentes para leer y escribir. Así, por ejemplo, el día del aniversario de la 
Madre fundadora de la orden que dirige el centro, la maestra contó a los niños su biografía y después les pidió que la escribieran. No todas las tareas requerían el mismo esfuerzo pero los niños las realizaban con ayuda de la maestra. Se usaron, además del método, otros materiales como periódicos y cuentos.

Los contenidos de enseñanza que más se trabajaron fueron: nombre y valor sonoro de las letras, escritura, identificación de palabras/lectura, función de la lectura y la escritura, léxico, familiaridad con los textos.

Ofrecemos a continuación un ejemplo de tarea de práctica multidimensional. Consistió en la escritura individual de la comida favorita de los niños.

(La maestra explica la ficha que van a realizar, y puesto que considera que es muy fácil, plantea a los niños otra tarea: cada uno debe escribir su comida favorita. La maestra intenta compaginar el método con otra tarea de escritura con funcionalidad social para despertar el interés de los niños y propiciar el uso de escrituras autónomas. Entrega a cada niño media cuartilla, que tiene escrito:

$<$ MI COMIDA FAVORITA

PRIMER PLATO

SEGUNDO PLATO

POSTRE>

M: Primero escribimos, arriba la fecha y el nombre. Cada niño escribe lo que a él le gusta comer, no vale copiarse.

N1: (a sus compañeros de mesa) si yo no he escrito la comida. (Pregunta a la M) ¿qué escribo?

M.: lo que te gusta a ti

(Los niños escriben concentrados, cada uno como sabe y puede. M se pasea por entre las mesas y apoya a los niños que lo necesitan).

N2: ¿Cómo se escribe mousse?

N3: se escribe mousse, yo lo he visto en el vasito.

M.: se escribe con dos eses.

N4: (escribe y habla con su compañera de mesa de lo que está escribiendo, copia de su compañera).

N2: ¿Con qué letra se escribe chocolate? 


\section{M: Con la ce hache}

N5: (tapa con la mano lo que está escribiendo para que no se lo vean los niños que están sentados a su lado).

\section{Aula C con prácticas de tipo instruccional}

En esta aula, los niños respondían a las demandas colectivas o individuales de la maestra. Eran demandas exigidas por la tarea y no solían intervenir de forma espontánea, ni interaccionar para trabajar, aunque sí intervenían de forma espontánea para preguntar dudas. La maestra procuraba que estuvieran callados, sentados en mesas individuales. Las opciones para romper la dinámica habitual debían estar justificadas, por ejemplo, sacar punta a un lápiz permitía a los niños levantarse de su sitio. El ambiente de la clase era tranquilo. La maestra intervenía para explicar, repetir, preguntar, poner ejemplos. Aprovechaba las ocasiones para provocar reflexión metalingüística, especialmente sobre el significado de las palabras.

Se observaron preferencias por el trabajo con el libro de texto y con el cuaderno de ejercicios. En él completaban el trabajo de escritura que el propio libro exigía: tareas simples como completar frases, escribir palabras, unir palabras con flechas, rodear palabras y colorearlas siguiendo consignas. No se provocó la escritura autónoma de textos ni se observaron iniciativas de los niños al respecto. A los alumnos más atrasados se les pedía que intervinieran en todas las tareas, controlando su aprendizaje y dándoles opciones de contribuir o participar.

Los contenidos trabajados en las tareas fueron: lectura-identificación de palabras, subrayado, escritura de palabras, caligrafía, léxico, morfología. Los materiales utilizados fueron el libro y los cuadernos de una editorial.

Para ilustrar el análisis, mostramos un ejemplo de tarea de práctica instruccional. Consistió en trabajar en el cuaderno de ejercicios de lenguaje. 
Son las 10:17 de la mañana. Los niños se disponen a realizar un ejercicio del cuaderno que demanda lo siguiente:

$<1$. Subraya y escribe las palabras que indican menor tamaño $>$ (En el texto aparecen palabras como hormiguita, faldita, lacitos).

$<2$. Escribe las palabras que corresponden a las que has subrayado sin que indiquen menor tamaño.

3. Rodea los, las, lo, la y fíjate en los nombres que acompaña.

4. Une con flechas cada animal con su casa>

El cuaderno de ejercicios marca las pautas de acción y los contenidos. No hay que realizar en ningún caso escritura autónoma, aunque sí lectura. El trabajo se repite con el fin de provocar un efecto de refuerzo sobre lo aprendido. Los niños saben qué se les pide y la corrección de errores es posterior a la tarea.

M: Voy a repartir el cuaderno de lengua que ya está corregido. Mientras termino de repartir vais a mirar si hay alguna falta. Los lápices con la punta bien afilada. Estamos en la página 43.

(...)

M: vamos a guardar el libro en la cajonera y luego me lo tenéis que dar para que lo corrija yo. Vais a sacar el cuaderno de lengua, que también me lo dais, después, para corregir.

$(\ldots)$

M: vamos a trabajar lo mismo que en el libro para reforzar. Lee, María.

N1 (leyendo): subraya y escribe las palabras que indican menor tamaño: hormiguita, faldita, lacitos...

M: (vuelve a leer lo mismo que la niña) ¿Qué será eso? Vamos a subrayar, ¿qué es subrayar? Niños: poner una rayita debajo

M: pues tenemos que escribirlas en horizontal. (Repite las palabras que quiere que subrayen). Pablo, ¿cómo empezamos nosotros?

N2: de izquierda a derecha.

N3: madre, mira hormiguita

M: muy bien. Vamos a dar envidia a todo el colegio cuando vean la letra. Lee, Manuel

N4: (lee) Escribe las palabras que corresponde a las que has subrayado sin que indique menor tamaño.

M. (explica) a hormiguita corresponde hormiga, esto significa que no es de menor tamaño.

(Los niños escriben las palabras en sus cuadernos)

M. Lee, Teresa.

N5 (lee) rodea los, las, lo, la

M.: fíjate en los nombres que acompaña. 
(Los niños realizan la tarea en silencio. Hacen comentarios cuando van terminando)

M.: lee, Ana

N6: (lee) Une con flechas cada animal con su casa

(Los niños unen con flechas: hormiguero con hormiga; abeja con colmena...)

M: ¿Por qué letra empieza colmena?

Niños: (a coro): por la ce

\section{Discusión}

Uno de los parámetros esenciales que se han de tener en cuenta en la enseñanza de la lengua escrita es la dinámica de participación e interacción social para provocar el aprendizaje (Vigotsky, 1973; Bruner, 1997). El Aula A, con prácticas situacionales, presenta una dinámica más participativa y colaborativa en el desarrollo de las tareas de lectura y escritura que el aula con prácticas instruccionales. En la primera se ofrece a los niños más oportunidades y mayores posibilidades de aprendizaje reflexivo, variado, social y contextualizado. La lectura y la escritura son consideradas actividades comunicativas, de práctica social, que conllevan una acción situada en un contexto (Bajtín, 1982), y provocan el uso y aprendizaje de géneros sociales (notas, cartas, recetas, noticias, etc, en esta etapa de la vida escolar) y no tanto de los géneros “escolares” propuestos dentro del formato único, "libro de texto". Sin embargo, en el Aula C, con prácticas instruccionales, la dinámica de la clase lleva a tareas más simples, con instrumentos y materiales únicos. Todo ello conduce a un aprendizaje más escolar, menos creativo y menos adaptado a las posibilidades diferenciadas de cada persona. En el Aula B, con prácticas multidimensionales, la dinámica es variada e incluye tareas de diferente complejidad, por lo que se adoptan medidas y dinámicas mixtas respecto a los otros dos tipos de prácticas, más alejados entre sí.

Respecto a las tareas propuestas en las aulas observadas, en el Aula A, con prácticas situacionales, los niños pueden participar en la producción de los textos, discutir, intercambiar, y prestarse ayuda. En el Aula C, con prácticas instruccionales, no se producen 
textos libres, ni autónomos, ni colectivos; se trabaja con palabras, por lo que no es necesario reflexionar sobre las características textuales. No hay oportunidad de participar de forma autónoma ni de cooperar con los compañeros. En el Aula B, con prácticas multidimensionales, sin embargo, cuando los niños producen textos intercambian, preguntan y se ayudan creando una dinámica variada. Por tanto, en las aulas A y B, con prácticas situacionales y multidimensionales respectivamente, se aprecian usos de la lengua escrita más complejos y de mayor nivel de exigencia cognitiva. Algunas de las propuestas didácticas de mayor relevancia en nuestro contexto (Fons, 1999; Bigas y Correig; 2000; Tolchinsky, 1993, 2001) defienden parámetros de enseñanza y aprendizaje semejantes a los que ofrecen el Aula A y en último término el Aula B.

Los contenidos abordados en el Aula A, con prácticas situacionales, son más contextualizados socialmente, más variados y complejos y hacen que los niños estén predispuestos a participar en retos cognitivos altos y en tareas de comunicación de mayor nivel. Se trata de aprender a leer y a escribir y de que las habilidades y estrategias que se aprenden estén en relación con el contexto real. Sin embargo, los contenidos del Aula C, con prácticas instruccionales, son más rutinarios y conducen a un aprendizaje alejado de las situaciones sociales de comunicación. Suelen estar relacionados con habilidades de bajo nivel, de las llamadas de tipo periférico (desciframiento, relación-sonido-grafía). Los contenidos del Aula B, con prácticas multidimensionales, son variados y van desde lo más rutinario del método de enseñanza de la lengua escrita más formalizado hasta los más complejos de las tareas de producción de textos. Por tanto, las prácticas de tipo situacional, y en parte las multidimensionales, provocan en el aula situaciones que alientan a ser realizadas en colaboración y a partir de lo que cada sujeto puede desarrollar. En el Aula C, con prácticas instruccionales, el trabajo sobre el código constituye casi exclusivamente el eje de acceso al mundo de lo escrito y el respeto al orden establecido en el acceso al código no permite aprovechar las situaciones emergentes para 
enseñar y aprender. Dedicar mucho tiempo al desciframiento supone no emplearlo para tareas de escritura colectiva o autónoma, a redactar textos complejos, a leer textos diversos, lo que acarrea el riesgo de ofrecer menos oportunidades para el desarrollo de la alfabetización. Nuestro trabajo de análisis corrobora la hipótesis de que la alfabetización es una tarea muy compleja (Camps, 1994; Teberosky y Tolchinsky, 1995; Solé et al., 2005) y muestra que en las aulas con prácticas de tipo situacionales se escriben y se leen textos que conllevan mayor dificultad y que tienen que ver con la realidad sociocultural de la escuela (Bruner, 1997; Fons, 1999).

Por otro lado, las tareas complejas de lectura y escritura necesitan de una mayor ayuda del docente en su desarrollo, lo que comporta una consideración de la alfabetización como un conjunto de retos para los que es necesario guiar el aprendizaje (Wharton-MacDonald et al., 1998; Pressley et al., 2001; Mercer, 1997). En el Aula A, con prácticas situacionales, la corrección de los errores o la mejora de las producciones se hacen con los niños. Se enseña a partir del error a buscar la solución correcta. Se construyen los textos de forma colectiva y se avanza en consonancia con lo que se sabe. En el Aula C, con prácticas instruccionales, la corrección realizada sin la presencia de los niños (corrección de cuadernos) no permite su utilización como instrumento de aprendizaje. En el aula B, con prácticas multidimensionales, la maestra corrige delante de los niños y les ayuda a reflexionar sobre sus errores. La promoción de actividades de alto nivel y la creación de condiciones de participación y ayuda que autores como Pressley et al. (2001) relacionan con un mayor nivel de eficacia docente se ha observado más en las aulas con prácticas situacionales y multidimensionales. En estas aulas es también más habitual que se escriba o se lea para comunicar al tiempo que se reflexiona sobre lo que se hace (Camps, 1994; Camps y Ribas, 2000).

A su vez, en nuestro estudio se aprecia que en las aulas con prácticas situacionales y multidimensionales se llevan a cabo tareas variadas, semejantes a las que se abordan desde 
las perspectivas analítica y analítico-sintética a las que se refiere Castells (2006, 2009a). Los materiales que se emplean van más allá de la estandarización que supone una publicación concreta ya que dichas tareas requieren otro tipo de materiales más relacionados con la vida de los niños y su entorno (periódicos, folletos, libros de conocimientos, modelos de cartas, etc).

El análisis detallado de las tareas de lectura y/o escritura realizadas en estas tres aulas permite concretar algunos de los rasgos de los tipos_de prácticas definidos por González Riaño, Buisán, y Sánchez (2009) y caracterizados con más detalle por Fons y Buisán (en este número). Tolchinsky, Ribera y García-Parejo (también en este número) señalan que no existe relación estadísticamente significativa entre estos tres tipos de prácticas y los logros de aprendizaje de los niños. Sin embargo, en la evaluación de los logros de los niños a los que se hace referencia en ese estudio no se llevaron a cabo tareas que mostraran sus saberes disponibles en situaciones de autonomía escritora. En este trabajo se ha visto que, al menos en estas tres aulas, este aspecto es uno de los que más diferencias marca entre las prácticas de tipo situacional, las prácticas de tipo multidimensional y las prácticas de tipo instruccional y creemos es conveniente tenerlo en cuenta a la hora de analizar la relación entre prácticas docentes y los resultados de aprendizaje, máxime cuando se ha identificado como uno de los indicadores de eficacia en la enseñanza de la lectura y la escritura (Pressley et al., 2001). Es importante considerar también cómo son los itinerarios formativos de los niños, yendo más allá del análisis de las tareas realizadas en las aulas durante un año escolar. En dichos itinerarios se producen cambios de maestra y por tanto de tipos de prácticas. Habría que dilucidar qué condiciones son esenciales en cada periodo y cómo influyen los cambios en el itinerario formativo del alumnado.

Por otro lado, el análisis de lo que ocurre en más aulas en diferentes contextos socioculturales y sociolingüísticos puede arrojar luz sobre otros fenómenos, como la influencia de la L1 o la 
L2, de las ayudas familiares a los niños y a la maestra o de los diferentes estilos de aprendizaje de los niños como variables en sus progresos o en las condiciones favorables de aprendizaje.

Las categorías empleadas en nuestro estudio para analizar las observaciones de aula remiten a una concepción de la enseñanza de la lengua escrita que va más allá de los métodos, los cuales se centran en el abordaje del código y no plantean otras variables esenciales en la alfabetización como la organización del aula y su dinámica, el contexto creado, los materiales que se ponen a disposición de los niños y, por tanto, la concepción sobre lo que es leer y escribir que tienen las docentes.

Los resultados de este estudio sugieren que en aulas en las que se lleven a cabo prácticas situacionales y mutidimensionales se dan más características que según distintos autores han sido señaladas como más efectivas para el aprendizaje.

Por último, hay que destacar que la formación del profesorado se puede ver beneficiada por esta aproximación a las aulas como contextos en los que ocurren fenómenos diversos y múltiples sobre los que reflexionar. Los rasgos que caracterizan los tipos de prácticas, corroborados por la observación directa de las situaciones de aula, se convierten en un posible modelo, abierto y flexible, para que la formación de los maestros se aproxime a las necesidades reales del sistema educativo y a las maneras de enseñar la lengua escrita que se muestran más efectivas. 
Notas

\footnotetext{
${ }^{1}$ Líneas de trabajo: formación de maestros; enseñanza inicial de la lengua oral y escrita; formación de maestros en contextos plurilingües y multiculturales.

${ }^{2}$ Líneas de trabajo: evolución de las competencias letradas en Educación Infantil y Primaria.

${ }^{3}$ Líneas de trabajo: análisis de situaciones de aula de Educación Infantil y Primaria; Necesidades organizativas y formación del profesorado; Recursos y tecnologías educativas
}

${ }^{4}$ Nos referimos al Proyecto APILE (SEJ: 2006/05292), sobre el que es posible encontrar más información en Sánchez y Alonso-Cortés y Barrio, Sánchez y Díaz (en este volumen)

${ }^{5}$ Consideramos tarea el fragmento de la actividad que tiene una finalidad escolar y está enmarcada entre un principio y un fin.

${ }^{6}$ De las observaciones de aula resultaron dos clases de datos, registrados respectivamente en las tablas de tareas y las tablas de interacciones (ver Sánchez y Alonso-Cortés, en este volumen). Para nuestro estudio nos centraremos en las tablas de tareas.

${ }^{7}$ Para la transcripción se ha seguido las siguientes pautas: las intervenciones de la maestra se señalan con la letra $\mathrm{M}$, las de los niños con la letra $\mathrm{N}$ seguida de una numeración para cada niño que intervenga. En el caso de que haya alguna referencia a niños, los nombres utilizados son ficticios. Se transcriben literalmente las intervenciones y en los casos en que es necesario se incluye al lado la traducción al castellano. Los fragmentos de texto que se corresponden con lengua escrita se reproducen entre los símbolos $<>$. Cualquier información contextual relevante se introduce entre paréntesis (). 


\section{Referencias}

Altava, V.; Gimeno, F.; Pérez, I.; Ríos, I.; Gallardo, I. M. (2006). El análisis de las situaciones de aula como instrumento de la formación del profesorado. En A. Camps (coord.) Diálogo e investigación en las aulas, (pp. 263-285). Barcelona: Graó.

Bajtín, M. (1982). La estética de la creación verbal. México: Siglo XXI.

Barrio, J.L; Sánchez, S. \& Díaz, L. (en este volumen) Repercusiones de las metodologías de investigación en la construcción de conocimiento didáctico: una investigación sobre el aprendizaje inicial de la lengua escrita

Bereiter, C. \& Scardamalia, M. (1992). Dos modelos explicativos de los procesos de composición escrita. Infancia y Aprendizaje, 58, 43-64.

Bigas, M. \& Correig, M., eds. (2000) Didáctica de la lengua en la educación infantil. Madrid: Síntesis.

Bruner, J. (1997). La educación, puerta de la cultura. Madrid: Visor

Camps, A. (1994). L'ensenyament de la composició escrita. Barcelona: Barcanova

Camps, A. \& Ribas, T. (2000). La evaluación del aprendizaje de la composición escrita en situación escolar. Madrid: CIDE, MEC

Castells, N. (2006). L'aprenentatge de la lectura inicial: una aproximació als coneixements dels infants $i$ a les estratègies d'ensenyament, Tesis Doctoral (Universitat de Barcelona), Recuperada en marzo de 2010 en: http://www.tesisenred.net/TDX-0129107-124121.

Castells, N. (2009a). La problemática de los métodos de enseñanza de la lectura: ¿qué sabemos en este momento? Aula de innovación educativa, 179, 29-32.

Dolz, J. \& Plane, S. (2008) Formations des enseignants et enseignement de la lecture et l'écriture. Namur (Bélgica): Presses Universitaires de Namur.

Fernández, P. (2007). "La mediación en la adquisición inicial de la escritura de diferentes géneros discursivos" en La magia de las letras. El desarrollo de la lectura y la escritura 
en la Educación Infantil y Primaria (pp.119-154). Madrid: Secretaría General Técnica, Ministerio de Educación y Ciencia.

Ferreiro, E. \& Teberosky, A. (1979). Los sistemas de escritura en el desarrollo del niño. México: Siglo XXI

Fons, M. (1999). Llegir i escriure per viure. Barcelona: La Galera

Fons, M. \& Buisán, C. (en este volumen) Entrevistas y observaciones de aula: otra mirada a los perfiles de prácticas docentes

González, X.A., Buisán, C. \& Sánchez, S. (2009). Las prácticas docentes para enseñar a leer y escribir. Infancia y Aprendizaje, 32, 2, 153-169.

Graves, D. (1983). Didáctica de la escritura. Madrid: Morata -MEC

Hayes, J.R. \& Flower, L. S. (1986) Writing research and the writer. American Psychologist, 41, 1106-1113.

Mercer, N. (1997) La construcción guiada del conocimiento. Barcelona: Paidós.

MEC (2006) PIRLS 2006. Estudio Internacional de progreso de la comprensión lectora. Informe español. Madrid: Ministerio de Educación y Ciencia.

Morrow, L. M. \& Schickedanz, J.A. (2006). The relationships between sociodramatic play and literacy development, en D.K. Dickinson \& S.B. Neuman (Eds). Handbook of early literacy research. Vol II., cap. 20, (pp. 269-280). New York: Guilford Press.

Morrow, L.M \& Gambrell, L.B. (2006). Literature-based instruction in the early years. En D.K. Dickinson \& S.B. Neuman (Eds.) Handbook of early literacy research. Vol I. New York, Guilford Press, cap. 23, pp. 348-360.

OCDE (2006). Informe PISA. Programa Internacional para la Evaluación de Estudiantes.

Olson, D.R. (2004). Alfabetización y educación: tres problemas para una teoría de la lectoescritura. Infancia y aprendizaje, 27 (2) 155-161. 
Pressley, M.; Wharton-Macdonald, R.; Allington, R. Collins Block, C., Morrow, L., Tracey, D., Baker, K.; Brooks, G., Cronin, J., Nelson, E., Woo, D (2001). A Study of Effective First-Grade Literacy Instruction. Scientifics Studies of Reading, 5(1), 35-58.

Sánchez, S. \& Alonso-Cortés, M.D. (en este volumen) Aprendizaje inicial de la lengua escrita: prácticas docentes y conocimientos de los alumnos.

Solé, I.; Mateos, M.; Miras, M.; Martín, E.; Castells, N.; Nuevas, I.; \& Graciá, M. (2005). Lectura, escritura y adquisición de conocimientos en Educación Secundaria y Educación Universitaria. Infancia y Aprendizaje, 28,(3), 329-347.

Teberosky, A. \& Tolchinsky, L. (1995). Más allá de la alfabetización. Buenos Aires: Santillana.

Tolchinsky, L. (1993). Aprendizaje del lenguaje escrito. Barcelona: Anthropos

Tolchinsky, L. (2001). Escribir y leer a través del currículum. Barcelona: ICE-Horsori

Tolchinsky, L; Ribera, P. \& García-Parejo, I. (en este volumen) Conocimientos iniciales y logros de los alumnos en el aprendizaje de la lectura y la escritura.

Vigotsky, L.S. (1973). Pensamiento y lenguaje. Buenos Aires: La Pléyade.

Wells, G. (1998). Aprender a leer y escribir. Barcelona: Laia

Wells, G. (2000): Indagación dialógica. Hacia una teoría y una práctica socioculturales. Barcelona: Paidós.

Wharton-McDonald, R., Pressley, M. \& Hampston, J. (1998). Outstanding literacy instruction in first grade: Teacher practices and student achievement. Elementary School Journal, 99, 101-128. 